\title{
Variability in Management Practices and Surgical Decision Making in Spinal Tuberculosis: An Expert Survey-Based Study
}

\author{
Kaustubh Ahuja, Tushar Gupta, Syed Ifthekar, Samarth Mittal, Gagandeep Yadav, Pankaj Kandwal \\ Department of Orthopaedic Surgery, All India Institute of Medical Sciences, Rishikesh, India
}

\begin{abstract}
Study Design: Electronic survey-based study.
Purpose: The aim of the study was to objectively review the variability in the prevailing treatment protocols and surgical decision making in the management of patients with spinal tuberculosis (TB) among spine surgeons with expertise in spinal TB across the country.

Overview of Literature: A lack of good-quality evidence, ambiguities in the national spinal TB guidelines, and the demand for early rehabilitation and a better quality of life in patients with spinal TB has led to the emergence of various gray zones in the management of spinal TB.

Methods: Seventeen fellowship-trained spinal TB experts representing different geographical regions of India completed an online survey consisting of questions pertaining to the conservative management of spinal TB (antitubercular therapy) and 30 clinical case vignettes including a wide spectrum of presentations of spinal TB with no or minimal neurological deficit. The variability in the responses for questions and case wise variability with respect to surgical decision making was assessed using the index of qualitative variation (IQV). The average tendency to operate (TTO) was calculated for various groups of respondents.

Results: High variability was observed in all questions regarding conservative spinal TB management (IQV >0.8). Among the 30 case vignettes, 14 were found to have high variability with respect to surgical decision making (IOV >0.8). With respect to levels of fixation, all but two cases had poor or slight agreement. Younger age and practice in a government or tertiary care teaching hospital were factors associated with a higher TTO.

Conclusions: Significant variability was detected in treatment practices for the management of spinal TB among experts. Most of the case vignettes were found to have significant heterogeneity with respect to surgical decision making, which reflects a significant lack of consensus and lacunae in literature.
\end{abstract}

Keywords: Spinal tuberculosis; Health care surveys; Consensus; Spinal fusion

\section{Introduction}

Tuberculosis (TB) of the spine is a complex spinal dis- order with a wide spectrum of complications that have a significant effect on the patient's quality of life. Of these complications, neurologic deficit is the most disastrous,

Received Nov 2, 2020; Revised Nov 30, 2020; Accepted Dec 1, 2020

Corresponding author: Pankaj Kandwal

Department of Orthopaedic Surgery, All India Institute of Medical Sciences, Rishikesh-249203, Uttarakhand, India

Tel: +91-8475000282, Fax: +91-0135-2452940, E-mail: pankaj.orth@aiimsrishikesh.edu.in 
adding considerably to the morbidity or mortality associated with spinal TB. Management strategies for spinal TB have undergone a continuous evolution since the prechemotherapeutic era, concurrent with the advancement in drug therapy and surgical techniques. One of the landmark events in the historical evolution of the management of spinal TB was the introduction of the idea of a "middle path" regimen by professor Tuli [1] in 1975. This regimen remains the most widely practiced treatment strategy and forms the basis for most guidelines on the management of spinal TB. Although it is highly effective, this treatment strategy is associated with a considerable period of bed rest, which contributes significantly to disability-adjusted life-years apart. Moreover, it is also associated with an element of uncertainty with respect to surgical decision making. In contrast to a predominantly conservative treatment strategy, surgical management of spinal TB results in early mobilization and rehabilitation, better short-term clinical outcomes that ensure an early return to work, and better kyphosis correction when compared with conservative management [2]. The advent of video-assisted thoracoscopic surgery and minimally invasive surgery for spinal $\mathrm{TB}$ in contrast to the highly invasive anterolateral surgical technique has made surgery a low-risk and high-reward option for the management of spinal TB [3-6]. In the light of these recent advances, some surgeons have lowered their threshold for surgery in spinal $\mathrm{TB}$, representing the dynamism in the evolution of surgical management of the disease. This assemblage of conventional middlepath followers and surgeons after early surgery has led to inhomogeneity in the management strategy, more so in patients with no or minimal neurologic deficit.

Index TB guidelines were introduced in 2016 to provide an evidence-based blueprint for the uniform diagnosis and chemotherapeutic and surgical management of spinal TB across the country [7]. However, because of a lack of adequate evidence, such as the role of biopsy in spinal TB and indications for surgery in spinal TB without neurologic deficit, there were a number of ambiguities in several discussion points. Thus, the authors of the current article sought to objectively review the prevailing treatment protocols and their variability among spine surgeons with expertise in the management of spinal TB across the country by performing a survey with preset questions and case vignettes focusing on the prevalent controversies in spinal TB management.

\section{Materials and Methods}

The authors performed an electronic survey-based crosssectional study to evaluate prevailing treatment practices among experts in spinal TB across the country. The study was approved by the institutional review board of All India Institute of Medical Sciences, Rishikesh (IRB no., AIIMS/IRB/19/895). Informed consent from individual patients was not taken because of retrospective and survey-based study design with blinding of all patient related information. The national directory for spine surgeons in the country was sought, and participants were selected for the study only if they fulfilled the following criteria: (1) five or more publications pertaining to spinal TB in indexed journals, (2) serving as chairpersons or active members of various national and international spine societies, (3) actively involved in managing at least 50 spinal TB patients annually, and (4) fellowship trained with at least 8 years of experience in spine surgery. While selecting participants, the authors also sought to ensure a sample of experts representing different geographical regions of the country.

The survey was designed to have three sections. Section one consisted of questions on the demographic details of the participating surgeon and his or her clinical practice. Section two focused on questions related to conservative management of spinal $\mathrm{TB}$, whereas section three contained 30 clinical vignettes representing real cases from the outpatient department of a tertiary care hospital. We obtained well-informed consent from all participants, and their identities were hidden before including the clinicradiologic details of patients in the questionnaire.

In section one, we sought to identify demographic factors that might have an impact on the surgeon's decision making, such as age, experience, type of practice (private/ government), and number of spinal TB patients managed each year. The questions included in section two were carefully designed after a thorough search of the literature, with special emphasis given to the prevailing controversies on spinal TB management. The questionnaire was multiple choice with preset options and included questions about diagnostic modality used, role of biopsy in guiding the start of antitubercular treatment (ATT), duration of treatment before confirmation with magnetic resonance imaging (MRI), and ATT regimen used for conservative management of spinal TB. The third section contained 30 clinical vignettes representing patients with 


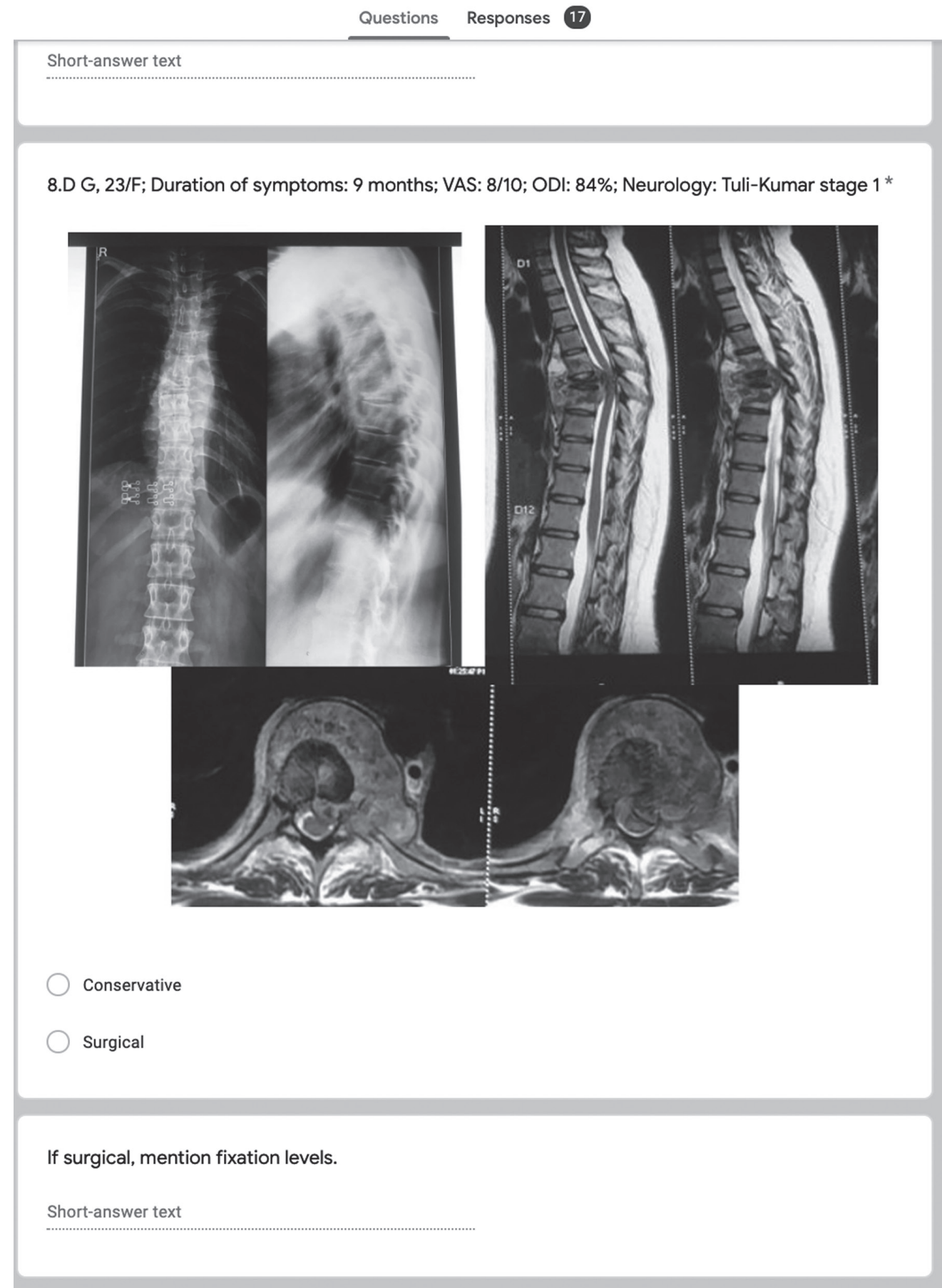

Fig. 1. Screen capture from the survey showing a vignette with dorsal spine tuberculosis.

spinal TB who had no or minimal neurologic deficit (Tuli and Kumar grade 0 and 1) [8]. Each vignette consisted of the patient's clinical information including age, sex, Oswestry Disability Index (ODI), Visual Analog Scale (VAS), and neurologic status (Tuli and Kumar grade 0 I or 1) and radiologic images including representative radiographs and an MRI composed of dedicated sagittal and axial sec- tions of the region of interest (Figs. 1,2). Special care was taken to include all regions of the spine affected by TB (C1 to S1) and various types of spinal TB based on the part of the affected vertebra (para-discal, central, posterior, and pan-vertebral). Due care was taken not to overcrowd each vignette with a lot of radiologic information and to include only important clinic-radiologic information to 
Questions Responses 17

Short-answer text

4 .B, 20/F; Duration of symptoms: 1 year; VAS for back pain: 10/10; ODI: 76\%; Neurology: TuliKumar stage 1

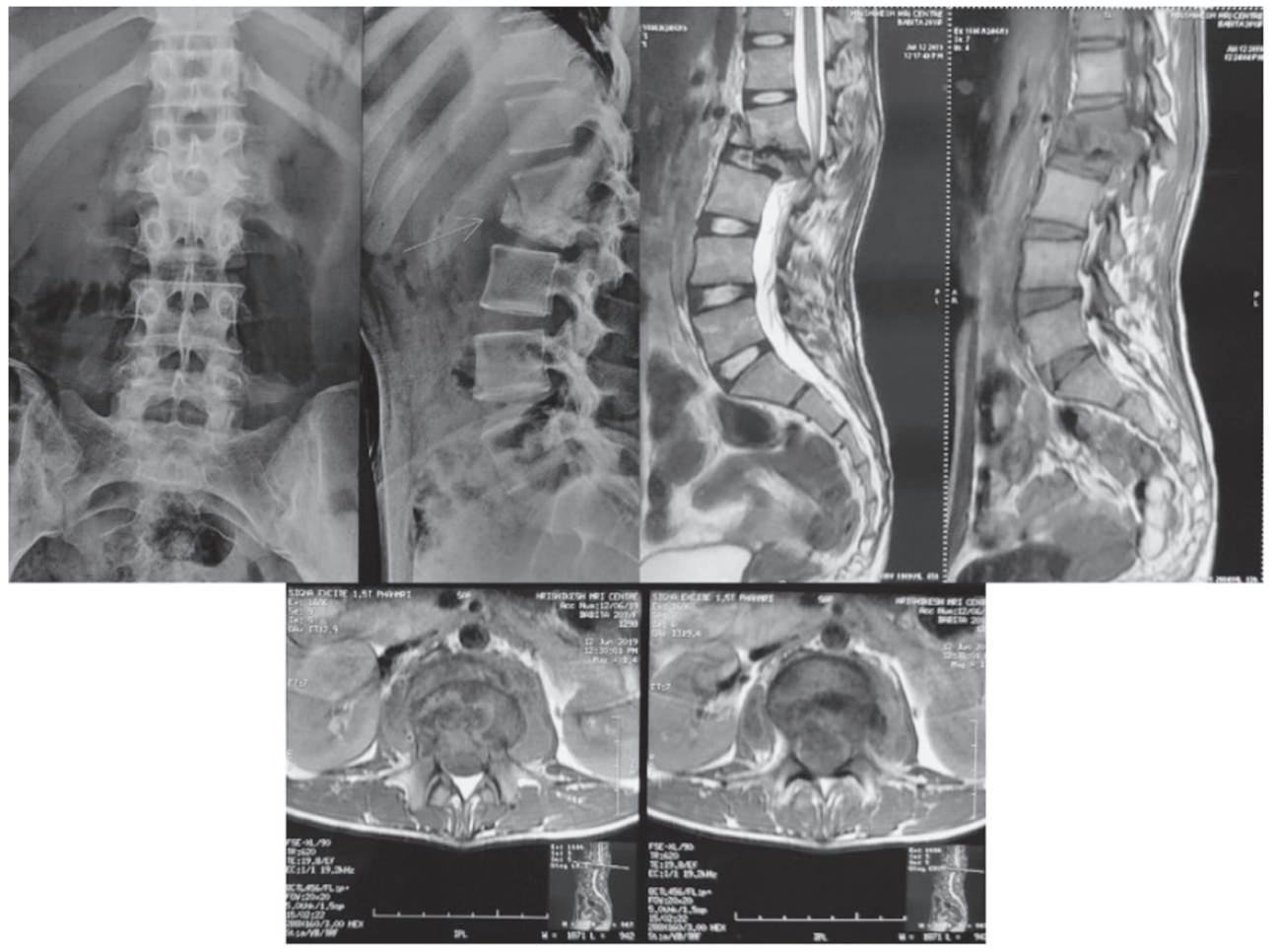

Conservative

Surgical

\section{If Surgical, mention levels of fixation.}

Short-answer text

Fig. 2. Screen capture from the survey showing a vignette with lumbar spine tuberculosis.

ensure the vignette was precise and accurate. For each vignette, participants were required to make a choice between conservative or surgical management based on the given information and mention the fixation levels for the cases for which surgery was the chosen option.

The electronic survey was created using Google documents and was sent to the selected participants via e-mail requests. A short description of the survey along with the 
Table 1. Demographic details of the respondents along with the TTO and $\kappa$-value for various demographical sub-groups

\begin{tabular}{|c|c|c|c|}
\hline Demographic factors & No. of respondents & TTO & $\kappa$-value \\
\hline Total respondents & 17 & $0.41 \pm 0.2$ & 0.25 \\
\hline \multicolumn{4}{|l|}{ Age (yr) } \\
\hline$<40$ & 6 & $0.58 \pm 0.2$ & 0.24 \\
\hline$>40$ & 11 & $0.35 \pm 0.2$ & 0.25 \\
\hline$p$-value & & 0.04 & \\
\hline \multicolumn{4}{|l|}{ Type of practice } \\
\hline Private & 9 & $0.32 \pm 0.2$ & 0.21 \\
\hline Government/tertiary care teaching & 8 & $0.50 \pm 0.1$ & 0.33 \\
\hline$p$-value & & 0.04 & \\
\hline \multicolumn{4}{|c|}{ No. of tuberculosis patients managed per year } \\
\hline$<100$ & 10 & $0.42 \pm 0.19$ & 0.26 \\
\hline$>100$ & 7 & $0.38 \pm 0.24$ & 0.24 \\
\hline$p$-value & & 0.35 & \\
\hline
\end{tabular}

Values are presented as number or mean \pm standard deviation, unless otherwise stated. Bold type is considered statistically significant.

TTO, tendency to operate

e-mail request and frequent reminders were sent to increase the number of responses. All responses were stored as data using Excel sheets and further analyzed with IBM SPSS ver. 25.0 (IBM Corp., Armonk, NY, USA). To evaluate the variability among responses, all responses from section two were analyzed using the index of qualitative variation (IQV). The IQV represents the ratio of the number of differences in the sample and maximum possible number of differences, with a score ranging from 0 to 1 , where 0 indicates no variability and 1 indicates maximum variability. For the analysis of section three, all clinical vignettes were individually analyzed for the IQV to evaluate concordance among respondents for surgical decision making. The vignettes were further categorized into the following three groups based on their IQV values: low variability-surgical (LVS), with an IQV less than 0.8 for surgical management; low variability-conservative (LVC), with an IQV less than 0.8 for conservative management; and high variability (HV), with an IQV greater than 0.8 . This was followed by a qualitative analysis for salient features for the identification of factors responsible for surgical inclination or HV among the respondents. In addition, we evaluated interrater agreement using the Fleiss kappa coefficient (k). A Fleiss kappa coefficient less than 0 represents "poor agreement," $\mathrm{k}$ between 0.01 and 0.20 represents "slight agreement," k between 0.21 and 0.40 represents "fair agreement," k between 0.41 and 0.60 represents "moderate agreement," $\mathrm{k}$ between
0.61 and 0.80 represents "substantial agreement," and k between 0.81 and 1.00 represents "almost perfect agreement." To analyze the levels of fixation, responses were evaluated in terms of upper instrumented vertebra (UIV) and lower instrumented vertebra (LIV) in the LVS and HV group vignettes; to determine interrater agreement with respect to levels of fixation, we calculated the Fleiss kappa coefficient for each case individually.

Furthermore, we calculated the tendency to operate (TTO) score for each surgeon. The TTO was defined as the arithmetic mean of the surgeon's responses for the 30 clinical vignettes (with 0 considered conservative and 1 considered surgical). A score closer to 0 signifies a higher likelihood of a surgeon choosing conservative treatment and vice versa. The respondents were further classified based on age ( $<40$ years, $>40$ years), type of practice (private, government) and number of spinal TB patients managed per year $(<100, \geq 100)$. We calculated and compared the mean TTO for each of these groups using an unpaired $t$-test and Mann-Whitney $U$-test for parametric and nonparametric data, respectively. We also calculated the $\kappa$-value within each group separately with respect to surgical decision. A $p$-value of less than 0.05 was considered statistically significant. 


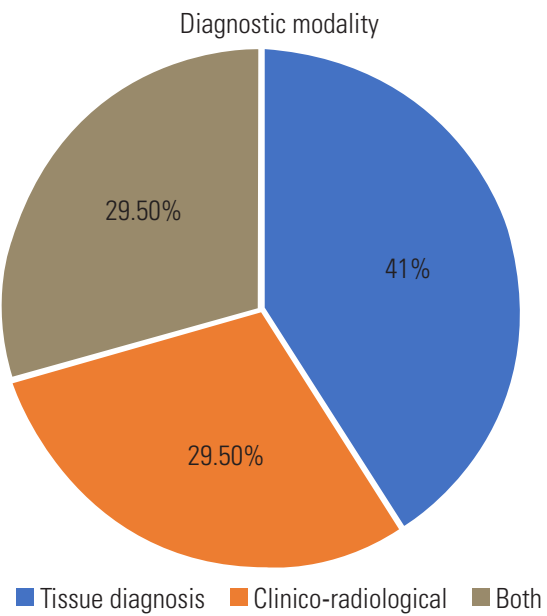

Fig. 3. Pie-chart showing the diagnostic modality of choice for all respondents.

\section{Results}

\section{Demographic details of respondents}

Of a total 24 invitations, 17 participants completed the survey (Table 1), all of whom were mal. Of the 17 respondents, 11 were older than 40 years, whereas six were younger than 40 years. With respect to the type of practice, nine respondents were working in private practice, whereas eight were working in a government or a tertiary care teaching hospital. Ten of the 17 respondents managed fewer than 100 spinal TB patients in a year, whereas seven managed more than 100 patients.

\section{Section two: multiple-choice questions on the con- servative management of spinal TB}

\section{1) Diagnostic modality}

The analysis of the responses indicated that $41 \%$ (7/17) of the respondents preferred "tissue diagnosis" as their diagnostic modality of choice (Fig. 3). Five respondents chose "clinico-radiological" and "both" options each. Of the 12 respondents who selected "tissue diagnosis" or "both," six chose to wait for the tissue diagnosis report before initiating ATT, whereas six others preferred starting ATT anyway. We found a high statistical variability among the responses to both of these questions $(\mathrm{IQV}=0.98, \mathrm{IQV}=1)$.

\section{2) Check MRI}

This question dealt with the minimum duration of ATT before performing a confirmatory contrast-enhanced MRI (Fig. 4). Of the 17 respondents, five chose "9 months,"

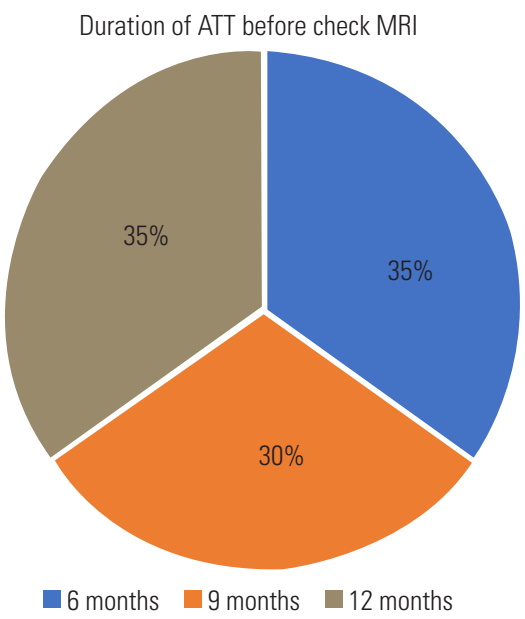

Fig. 4. Pie-chart showing the preferred duration of antitubercular therapy (ATT) before getting the check magnetic resonance imaging (MRI) done.

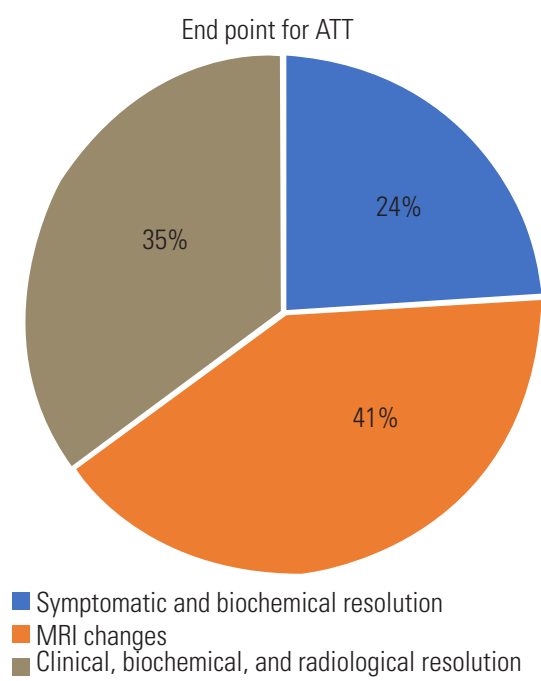

Fig. 5. Pie-chart showing the preferred investigation modality foe deciding the end point of antitubercular therapy (ATT). MRI, magnetic resonance imaging.

whereas six respondents chose " 6 months" and " 12 months" each. We found a significant variability among the responses (IQV=0.99).

\section{3) Endpoint for ATT}

Most respondents $(7 / 17,41 \%)$ preferred MRI findings of no enhancement and fatty infiltration as the endpoint for ATT (Fig. 5). Four respondents chose clinical and biochemical (erythrocyte sedimentation rate, C-reactive protein) resolution, whereas six preferred clinical, biochemical, and radiologic (all three) resolution before stopping ATT. The responses were found to have significant variability ( $\mathrm{IQV}=0.91)$. 


\section{4) ATT regimen}

This was a short-answer question in which the respondents were asked to mention the ATT regimen they followed in their practice. Six different responses were recorded, indicating a HV and poor consensus (Table 2).

\section{Section three}

Analysis of the information given in clinical vignettes indicated that the mean age of the included patients was $32.3 \pm 12.7$ years (range, $13-55$ years), with a mean duration of symptoms of $8.3 \pm 5.4$ months (range, $1-24$ months). The mean ODI and VAS score at the time of presentation was $76.7 \% \pm 14.2 \%$ and $7.8 \pm 1.6$, respectively. Table 3 shows the location-wise distribution of spinal TB. In the evaluation of IQV, 14 of 30 clinical vignettes

Table 2. Various ATT regimes followed by the respondents ( $n=17)$

\begin{tabular}{lc} 
ATT regime & No. of respondents \\
2 HRZE+10 HR & 5 \\
2 HRZE+10 HRE & 4 \\
\hline 2 HRZE+7 HRE+9 HR & 1 \\
\hline 3 HRZE+3 HRE+6 HR & 2 \\
\hline 4 HRZE+5 HRE & 1 \\
\hline 4 HRZE+14 HRE & 2 \\
\hline
\end{tabular}

ATT, anti-tubercular treatment; $H$, isoniazid; $R$, rifampicin; $Z$, pyrazinamide; $E_{\text {, }}$ ethambutol. were found to have $\mathrm{HV}$ and were categorized into the HV group. Of the remaining vignettes, six were classified as having LVS, whereas 10 were considered LVC. We did not find a statistically significant difference in the mean age, duration of symptoms, ODI, and VAS between the three groups. Furthermore, special features of the vignettes in the HV groups were analyzed qualitatively (Table 4).

Analysis of the overall interrater agreement revealed that the respondents were found to be in "fair" agreement, with a $\kappa$-value of 0.25 (95\% confidence interval, $0.15-0.36$ ). The respondents were further categorized into groups based on age, type of practice, and number of TB patients seen each year, and the agreement was fair within each $(<40$ years, 0.24 ; $>40$ years, 0.25 ; private, 0.21 ; government/tertiary care teaching hospital, $0.33 ;<100$ annual TB patients, 0.26 ; $>100$ annual TB patients, 0.24) (Table 1). Interrater agreement with respect to the levels of fixation was either poor or in slight agreement in all cases, except for one case of $\mathrm{C} 1-\mathrm{C} 2 \mathrm{~TB}$, for which the $\mathrm{k}$ was found to be 0.56 (moderate agreement) for UIV and one case of lumbar TB with a $k$ of 0.38 (fair agreement) for LIV (Table 5).

The mean TTO of all respondents was $0.41 \pm 0.2$. Surgeons younger than 40 years were found to have a significantly higher TTO as compared with the surgeons older than 40 years. In addition, surgeons who worked in a government setup or a tertiary care teaching hospital had a significantly higher TTO than those working in a private setup. There was no difference in TTO based on the number of spinal TB patients managed per year (Table 1).

Table 3. Details of vignettes over all and in three groups with comparison

\begin{tabular}{|c|c|c|c|c|c|}
\hline Variable & Total & LVS & $\mathrm{HV}$ & LVC & $p$-value \\
\hline No. of vignettes & 30 & 6 & 14 & 10 & \\
\hline Age (yr) & $32.3 \pm 12.7$ & $22.8 \pm 10.8(13-44)$ & $32.7 \pm 12.6(15-55)$ & $37.5 \pm 11.8(16-54)$ & 0.07 \\
\hline Duration of symptoms (mo) & $8.3 \pm 5.4$ & $8.5 \pm 3.5(3-12)$ & $8.2 \pm 5.4(1-24)$ & $8.5 \pm 6.9(1-24)$ & 0.98 \\
\hline Oswestry Disability Index & $76.7 \pm 14.2$ & $71.6 \pm 21.0$ & $79.3 \pm 13.6$ & $76.2 \pm 10.3$ & 0.51 \\
\hline Visual Analog Scale & $7.8 \pm 1.6$ & $8.5 \pm 1.9$ & $7.98 \pm 1.9$ & $7.4 \pm 0.8$ & 0.45 \\
\hline \multicolumn{6}{|l|}{ Location } \\
\hline Cervical & 2 & 0 & 1 & 1 & \\
\hline Cervico-dorsal & 1 & 0 & 1 & 0 & \\
\hline Dorsal & 9 & 2 & 5 & 2 & \\
\hline Dorso-lumbar & 7 & 2 & 2 & 3 & \\
\hline Lumbar & 8 & 1 & 4 & 3 & \\
\hline Lumbo-sacral & 3 & 1 & 1 & 1 & \\
\hline
\end{tabular}

Values are presented as number, mean $\pm S D$, or mean $\pm S D$ (range), unless otherwise stated.

LVS, low variability-surgical; HV, high variability; LVC, low variability-conservative; SD, standard deviation. 
Table 4. Salient features of vignettes in high variability group

Salient features of vignettes in high variability category

Multifocal contiguous $>2$ intervertebral disc spaces with destruction of the intervening body

Active compression over the cord, conus, or thecal sac

Pan-vertebral disease or facet joint involvement

Retropulsion and lateral subluxation (hard compression)

Distraction of the posterior elements or facetal subluxation/dislocation

Kyphosis more than $45^{\circ}$

Table 5. $\kappa$-values for LVS cases and HV cases for UIV and LIV

\begin{tabular}{|c|c|c|c|}
\hline Variable & Category & $\begin{array}{l}\text { Free-marginal kappa } \\
\text { (UIV) }\end{array}$ & $\begin{array}{l}\text { Free-marginal kappa } \\
\text { (LIV) }\end{array}$ \\
\hline \multirow[t]{6}{*}{ LVS cases } & Case 1 & 0.03 & 0.09 \\
\hline & Case 2 & 0.08 & 0.13 \\
\hline & Case 3 & 0.12 & 0.05 \\
\hline & Case 4 & 0.01 & 0.05 \\
\hline & Case 5 & 0.23 & 0.08 \\
\hline & Case 6 & 0.13 & 0.05 \\
\hline \multirow[t]{14}{*}{ HV cases } & Case 7 & -0.06 & 0.13 \\
\hline & Case 8 & -0.07 & 0.23 \\
\hline & Case 9 & 0.02 & 0.02 \\
\hline & Case 10 & 0 & 0.38 \\
\hline & Case 11 & 0 & 0.14 \\
\hline & Case 12 & 0.56 & -0.04 \\
\hline & Case 13 & 0 & 0.17 \\
\hline & Case 14 & -0.07 & 0.09 \\
\hline & Case 15 & 0 & 0 \\
\hline & Case 16 & -0.33 & -0.33 \\
\hline & Case 17 & -0.2 & -0.2 \\
\hline & Case 18 & -0.2 & -0.2 \\
\hline & Case 19 & -0.4 & -0.2 \\
\hline & Case 20 & -0.2 & -0.2 \\
\hline
\end{tabular}

LVS, Iow variability-surgical; HV, high variability; UIV, upper instrumented vertebra; LIV, lower instrumented vertebra.

\section{Discussion}

Spinal TB is the most common form of TB that affects bone and joints and is highly prevalent in India and surrounding countries. In the past 2 decades, India had the second highest number of publications on spinal TB [9]. Despite this abundant quantity of literature, good-quality randomized and multicenter nationwide evidence on spi- nal TB remains scarce. There are several reports of glancing lacunae in the available literature, such as the role of biopsy as the primary diagnostic modality, the use of therapeutic vacuum before histopathology or microbiology report, the most effective ATT regimen, and the factors to be taken into consideration before stopping ATT. Similarly, the indications for surgical management, especially in patients without any neurologic deficit, are ambiguous and highly subjective. In the present study, we sought to analyze the prevalent treatment practices and variability among spine surgeons who have expertise in the subject with respect to conservative and surgical management of spinal TB with the assistance of a nationwide survey consisting of questions regarding conservative management and clinical vignettes for surgical decision making. This is a validated methodological tool for evaluating prevalent health care practices and has been widely employed for several spinal conditions [10-15].

\section{Variability in conservative management}

We found significant variability in all questions regarding conservative management. The index TB guidelines suggest starting ATT on a clinic-radiologic basis in endemic areas, whereas they recommend biopsy for culture and drug susceptibility testing from the lesion wherever possible [7]. In the current study, 12 of the 17 respondents chose either "tissue diagnosis" or "both" (clinic-radiologic and tissue) as their diagnostic modality of choice. Moreover, half of these respondents chose to wait for the histopathology or microbiology report before initiating ATT. This might lead to a considerable therapeutic vacuum, especially in a venue with a high patient load, as there may be a significant delay in obtaining computed tomographyguided biopsy and subsequent test results. There was no clear consensus with respect to the duration of treatment before undergoing a check MRI for reviewing disease status. Index TB guidelines recommend performing check MRI films at 6, 9, 12, and 18 months to assess healing [7]. However, financial and logistical restrictions make following these recommendations highly impractical. The responses in the current study were equally divided between durations of 6,9 , and 12 months. The decision regarding the endpoint for ATT in spinal TB is highly controversial, and there is no clear consensus in the literature with respect to the modality used or the treatment duration. In the current study, seven respondents relied on MRI 
findings, such as lack of contrast enhancement and fatty infiltration, whereas six respondents were in favor of continuing ATT until clinical, biochemical, and radiologic investigations were all in favor of a healed disease. Rest relied on only clinical and biochemical healing and did not consider MRI for decision making. With respect to the ATT regimen, we observed significant variability on analyzing the responses. The index TB guidelines recommend 2 months of HRZE ( $\mathrm{H}$, isoniazid; $\mathrm{R}$, rifampicin; $\mathrm{Z}$, pyrazinamide; E, ethambutol) followed by 10 months of HRE; however, in the current study, only four of the 17 respondents were found to follow the recommendation [7].

\section{Variability in surgical management}

Based on the clinical vignettes, the variability in surgical decision making was also found to be high. Of a total 30 clinical vignettes, 14 were found to have highly variable responses ( $\mathrm{QQV}>0.8$ ), whereas six vignettes were found to have responses with low variability for surgical management $(\mathrm{IQV}<0.8)$. On further analysis, HV vignettes were found to have less than or equal to two whereas LVS vignettes were found to have more than two of the following features: pan-vertebral involvement, bilateral destruction, lateral or anteroposterior subluxation or dislocation of the facet joints (hard compression), multifocal contiguous involvement involving more than two-disc spaces with intervening body destruction, and moderate to severe kyphosis or retropulsion. The above-described factors may be responsible for instability, which has been described in the literature as an indication for fixation [16-18]. Overall and category-wise interrater agreement was found to be only "fair," which, again, represents a lack of consensus among surgeons. The lack of objective criteria to determine instability may be responsible for the high degree of variability in the surgical decision making in such cases. We further evaluated all of the vignettes that were classified as either LVS or HV for the agreement between respondents in terms of levels of fusion. Irrespective of the location of the disease, the interrater agreement was poor or slight for both the UIV and LIV in 19 of 20 cases each. One case with $\mathrm{C} 1-\mathrm{C} 2$ spinal TB had moderate agreement among respondents with respect to the UIV and most recommended $\mathrm{O}-\mathrm{C} 2$ fusion, whereas another case with L2-L3 spinal TB had moderate agreement with respect to the LIV. We found a scarcity of literature with respect to the length of instrumentation in spinal TB. Only a single retrospective study was located, which reported reduced time and blood loss with short fixation as opposed to better correction of deformity with long construct [19].

With respect to the TTO among the respondents, we attempted to identify demographic factors that might be responsible for affecting a surgeon's TTO. We found that age and type of practice had a significant bearing on the TTO. Younger surgeons (age $<40$ years) were found to have a significantly higher TTO than older surgeons (age $>40$ years). This finding may suggest a higher familiarity with modern surgical techniques and instrumentation as opposed to more conventional, older surgeons. It may also suggest that younger surgeons have a more open mind in contrast to more rigid, older surgeons. Apart from age, surgeons working in government facilities were found to have a significantly higher TTO when compared with a surgeon working in a private setup. This may be partly attributable to the greater complexity and severity of the cases that present to a government setup as compared with a private setup.

Most resident training programs and national guidelines on spinal TB are based on the revolutionary "middle path" regimen, as given by professor Tuli [1] in the early 1970s. This regimen spoke of spinal TB as a primarily medical disease to be managed with antitubercular therapy and bed rest, whereas surgery was reserved for patients who demonstrated no clinical or neurologic improvement or worsening on conservative management [8]. Other indications described in the regimen included posterior spinal disease, doubtful diagnosis, or instability [1]. The regimen was formulated for places with fewer resources and high patient load, based on the fact that most TB patients with paraplegia have poor general health, anemia, and cachexia with associated pulmonary TB, and a fraction of these patients start showing neurologic and clinical recovery on ATT, bed rest, and nutritious diet within 6-8 weeks $[17,20]$. The regimen proved to be highly effective and demonstrated good results in managing spinal TB during that period. However, it also subjected patients to a long period of bed rest, which contributed considerably to the preexisting morbidity and mortality in these patients. Ambulatory chemotherapy was subsequently introduced, which allowed patients on chemotherapy to mobilize in a rigid brace or a plaster cast, thus preventing complications associated with recumbency [21]. To compare conservative and surgical options, pooled data from a number of prospective multicenter clinical trials that took place 
in Hong Kong, Rhodesia, and South Africa indicated a significant superiority of the radical "Hong Kong" procedure to conservative treatment or debridement alone in short-term follow-up with respect to early fusion and faster resolution of abscesses and in long-term follow-up with respect to correction of kyphosis [2,22]. Since then, a number of advancements in surgical instrumentation, such as modern pedicle screw fixation with minimally invasive and video-assisted thoracoscopic techniques, has been associated with minimal intraoperative blood loss and tissue disruption, with good clinical and radiologic outcomes and early mobilization and rehabilitation after surgery for spinal $\mathrm{TB}$, thus resulting in a reduction in complications and lesser loss in disability-adjusted lifeyears [3-5]. However, there is a scarcity of evidence comparing modern surgical techniques with the more conservative middle path regime. With the improvement in financial resources and inexpensive information available through the Internet, there has been an increased demand for better functional and cosmetic outcomes in patients undergoing management for spinal TB. In summary, the lack of good-quality evidence, ambiguities in the national TB guidelines for spinal TB, and the demand for early rehabilitation and a better quality of life in patients with spinal TB has led to the emergence of various gray zones in the management of this disease. Thus, a combination of the above-described factors might be responsible for the variability observed in the present study.

We faced a number of limitations and difficulties in carrying out the present study. While analyzing feedback, we recognized the long length of the questionnaire was an important impediment in obtaining a higher number of responses. However, to encompass various prevailing controversies and the large spectrum of spinal TB presentations, we believe the length of the survey was justified. Another limitation was the small number of survey invitations and consequent responses. Nevertheless, due emphasis was placed on including spine surgeons with reasonable expertise in the management of spinal $\mathrm{TB}$ and generating a sample that was representative of various geographical regions of the country. The large number of clinical vignettes $(n=30)$ resulted in a total of 510 responses, which is a significant number when compared with other similar studies. Furthermore, several other controversial topics, such as multidrug resistance, were not included in this paper. We would also like to acknowledge the problem of not having a unified national TB study group, which led to a significant number of difficulties in carrying out a large, nationwide survey. Finally, we also recognize the lack of objectivity in defining practice patterns in relation to the management of spinal TB.

Despite these limitations, this study was able to bring forward significant lacunae in the literature and guidelines on spinal $\mathrm{TB}$, leading to significant variability in treatment strategies prevailing across the country. Such wide variability in the management of such a prevalent and complex spinal disorder can lead to confusion among patients and treating physicians. Through our research article, we would further like to stress the need to form national spinal TB study groups, restructure the national guidelines on spinal TB in consensus with representative spine surgeons, and obtain good-quality evidence in the form of randomized multicenter trials for the management of spinal TB.

\section{Conclusions}

We found HV in the treatment practices of spinal TB experts across the country with respect to various aspects of conservative management and surgical indications for spinal TB, particularly for patients with no or minimal neurologic deficit. Moreover, there was either poor or only slight agreement on the levels of fusion. Factors such as the age of the surgeon and type of practice were found to have a bearing on the surgeon's TTO. This study highlights the need for good-quality evidence, restructuring of national guidelines, and objective criteria for surgical decision making for the management of spinal TB.

\section{Conflict of Interest}

No potential conflict of interest relevant to this article was reported.

\section{Author Contributions}

Conception of the work: Kaustubh Ahuja; the acquisition, analysis, interpretation of data for the work: Kaustubh Ahuja, Tushar Gupta, Samarth Mittal, Pankaj Kandwal; drafting of the work: Kaustubh Ahuja, Pankaj Kandwal; design of the work: Tushar Gupta; drafting and revising the work: Syed Ifthekar, Gagandeep Yadav; and supervision: Pankaj Kandwal. 


\section{References}

1. Tuli SM. Results of treatment of spinal tuberculosis by "middle-path" regime. J Bone Joint Surg Br 1975;57:13-23.

2. Mak KC, Cheung KM. Surgical treatment of acute TB spondylitis: indications and outcomes. Eur Spine J 2013;22(Suppl 4):603-11.

3. Garg B, Kandwal P, Nagaraja UB, Goswami A, Jayaswal A. Anterior versus posterior procedure for surgical treatment of thoracolumbar tuberculosis: a retrospective analysis. Indian J Orthop 2012;46:165-70.

4. Jayaswal A, Upendra B, Ahmed A, Chowdhury B, Kumar A. Video-assisted thoracoscopic anterior surgery for tuberculous spondylitis. Clin Orthop Relat Res 2007;460:100-7.

5. Kandwal P, Garg B, Upendra B, Chowdhury B, Jayaswal A. Outcome of minimally invasive surgery in the management of tuberculous spondylitis. Indian J Orthop 2012;46:159-64.

6. Ito $\mathrm{M}$, Sudo $\mathrm{H}$, Abumi $\mathrm{K}$, et al. Minimally invasive surgical treatment for tuberculous spondylodiscitis. Minim Invasive Neurosurg 2009;52:250-3.

7. Ministry of Health and Family Welfare. Index-TB guidelines: guidelines on extra-pulmonary tuberculosis for India. Geneva: World Health Organization; 2016.

8. Tuli SM. Treatment of neurological complications in tuberculosis of the spine. J Bone Joint Surg Am 1969;51:680-92.

9. Wang Y, Wang Q, Zhu R, et al. Trends of spinal tuberculosis research (1994-2015): a bibliometric study. Medicine (Baltimore) 2016;95:e4923.

10. Peabody JW, Luck J, Glassman P, Dresselhaus TR, Lee M. Comparison of vignettes, standardized patients, and chart abstraction: a prospective validation study of 3 methods for measuring quality. JAMA 2000;283:1715-22.

11. Peabody JW, Luck J, Glassman P, et al. Measuring the quality of physician practice by using clinical vignettes: a prospective validation study. Ann Intern Med 2004;141:771-80.
12. Gould D. Using vignettes to collect data for nursing research studies: how valid are the findings? J Clin Nurs 1996;5:207-12.

13. Lonjon G, Grelat M, Dhenin A, et al. Survey of French spine surgeons reveals significant variability in spine trauma practices in 2013. Orthop Traumatol Surg Res 2015;101:5-10.

14. Debono B, Lonjon G, Galovich LA, et al. Indication variability in degenerative lumbar spine surgery: a four-nation survey. Spine (Phila Pa 1976) 2018;43:18592.

15. Cetin E, Senkoylu A, Acaroglu E. Assessment of variability in Turkish spine surgeons' trauma practices. Acta Orthop Traumatol Turc 2018;52:1-6.

16. Jain AK. Treatment of tuberculosis of the spine with neurologic complications. Clin Orthop Relat Res 2002;(398):75-84.

17. Jain AK, Kumar J. Tuberculosis of spine: neurological deficit. Eur Spine J 2013;22(Suppl 4):624-33.

18. Rajasekaran S, Kanna RM, Shetty AP. Management of thoracolumbar spine trauma: an overview. Indian J Orthop 2015;49:72-82.

19. Liu Z, Zhang P, Zeng H, Xu Z, Wang X. A comparative study of single-stage transpedicular debridement, fusion, and posterior long-segment versus shortsegment fixation for the treatment of thoracolumbar spinal tuberculosis in adults: minimum five year follow-up outcomes. Int Orthop 2018;42:1883-90.

20. Tuli SM. Historical aspects of Pott's disease (spinal tuberculosis) management. Eur Spine J 2013;22(Suppl 4):529-38.

21. Rajasekaran S, Soundararajan DC, Shetty AP, Kanna RM. Spinal tuberculosis: current concepts. Global Spine J 2018;8(4 Suppl):96S-108S.

22. Controlled trial of short-course regimens of chemotherapy in the ambulatory treatment of spinal tuberculosis: results at three years of a study in Korea: twelfth report of the Medical Research Council Working Party on Tuberculosis of the Spine. J Bone Joint Surg Br 1993;75:240-8. 